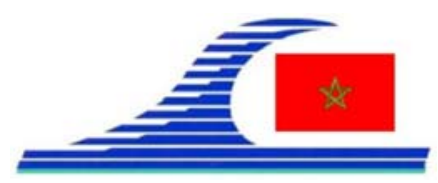

Conférence Méditerranéenne Côtière et Maritime

EDITION 2, TANGER, MAROC (2011)

Coastal and Maritime Mediterranean Conference

Disponible en ligne - http://www.paralia.fr - Available online

\title{
Evaluation de l'acceptabilité environnementale des sédiments de dragage en tant que matériaux alternatifs : Application du guide méthodologique
}

\author{
Nor Edine ABRIAK ${ }^{1}$, Raouf ACHOUR ${ }^{1}$, Rachid ZENTAR ${ }^{1}$ \\ 1. Ecole des mines de Douai, 941, rue Charles Bourseuil, 59508 Douai Cedex, France. \\ nor-edine.abriak@mines-douai.fr ; raouf.achour@mines-douai.fr ; rachid.zentar@mines-douai.fr
}

\section{Résumé :}

Dans la logique du développement durable, la valorisation des sédiments de dragage dans des conditions environnementales maîtrisées est nécessaire afin de réduire l'incidence globale liée à l'utilisation de ce matériau en technique routière. En application de ces conditions, la construction routière peut représenter une filière pour la valorisation de ces matériaux. En 2008, le volume de sédiments dragués en France (métropolitaine et outre-mer compris) représente un total de $37,4 \mathrm{Mm}^{3}$, soit une légère augmentation de 5\% par rapport au volume enregistré en 2007 (CETMEF, 2010).

La dissémination des différents contaminants contenus dans les matériaux de dragage présente un risque sur l'environnement. Pour cela l'adaptation du guide méthodologique "acceptabilité de matériaux alternatifs en techniques routières, évaluation environnementale" du MEDDTL en action et en essais est une démarche importante afin de bien identifier les sédiments de dragage et les classer selon des niveaux environnementaux.

Mots clés : Sédiments portuaire - Dragage - Matériaux alternatifs - Pollution lixiviation - Evaluation environnementale

\section{Introduction}

En France, la consommation des granulats présente 376 millions de tonnes de granulats provenant de gisement terrestres (UNPG, site web) provenant essentiellement de gisement terrestre. Devant les difficultés croissantes d'accès aux gisements terrestres, les producteurs de granulats diversifient leurs ressources, notamment par les granulats marins (sédiments marins). Ces matériaux extraits en mer possèdent des caractéristiques semblables à celles des granulats de roches meubles extraits de carrières terrestres. Ils sont donc une ressource complémentaire, en particulier pour les régions littorales et les grands centres urbains qui peuvent être desservis par voie d'eau. De plus avec la raréfaction de granulats provenant des carrières, les déblais de dragage peuvent devenir une nouvelle source des matériaux (DUBOIS et al, 2009).

La gestion des matériaux de dragage présente aujourd'hui un vrai problème en raison des fortes quantités de sédiments chaque année. Cette gestion est complexe, évolutive, et demande des moyens importants (BOUTIN, 2000). 
La connaissance de la Mer :

un vecteur du développement durable en Méditerranée

Cette gestion dépend de la nature et du degré de pollution des sédiments et les méthodes envisagées pour le traitement. Afin d'évaluer le potentiel d'utilisation des sédiments marins en construction routière, l'objectif est de classer les sédiments suivant un niveau environnemental précis et ensuite vérifié si le sédiment pourrait être utilisé en technique routière selon le guide méthodologique "acceptabilité de matériaux alternatifs en techniques routières, évaluation environnementale" du MEDDTL (SETRA, 2011).

Ce guide méthodologique s'adresse principalement aux professionnels des travaux publics et aux industriels qui souhaitent étudier les possibilités de valorisation, en technique routière, des déchets qu'ils détiennent ou qu'ils produisent. Par ailleurs, il reste à établir un guide d'application pour l'utilisation des sédiments en technique routière.

Dans notre étude nous allons appliquer le guide méthodologique et mettre en œuvre cette méthodologie qui vise à fournir une démarche d'évaluation de l'acceptabilité environnementale des matériaux alternatifs élaborés à partir de déchets (sédiments marins de port de Dunkerque bassin Est DARSE 6 "FREYCINET 12 et 13") destinés à être utilisés en technique routière.

\section{Etude méthodologique d'évaluation environnementale}

Le guide méthodologique présente une démarche permettant de valider l'utilisation d'un matériau alternatif pour un usage routier ne disposant ni de guide d'application ni de guide référence. Un déchet peut donc suivre un processus d'élaboration et devenir par la suite un matériau alternatif puis un matériau routier, ce processus fait l'objet d'une évaluation environnementale pour savoir si un matériau alternatif élaboré à partir des sédiments marins, peut être utilisé au sein d’un matériau routier (figure 1).

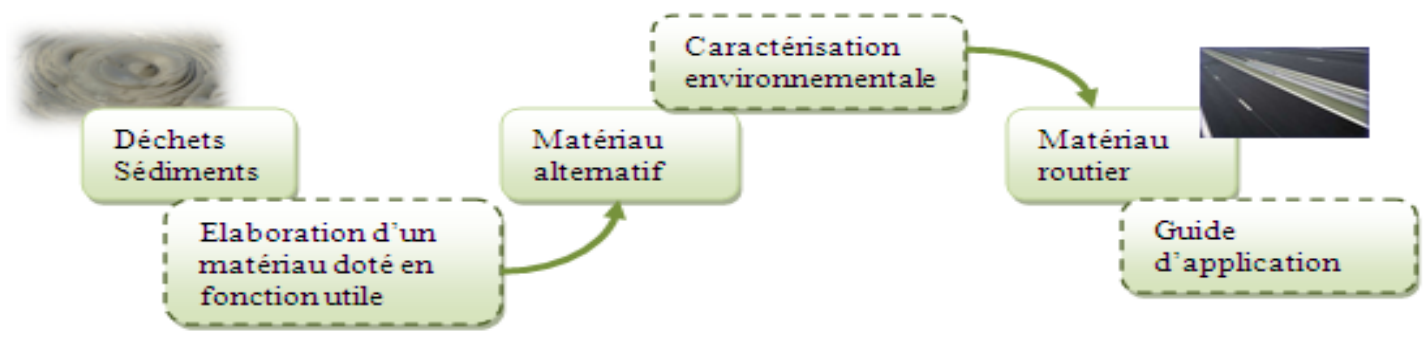

Figure 1. Processus d'évaluation environnementale pour une valorisation en technique routière.

A la pratique de ce processus, il faut s'assurer que le matériau alternatif, proposé pour l'utilisation en technique routière présente des caractéristiques géotechniques, mécaniques et hydrauliques conformes aux normes d'usage en techniques routières. La démarche du processus d'évaluation environnementale est définie par une méthodologie de détermination du relargage des éléments des sédiments vers l'eau, dans des conditions spécifiées d'utilisation ou de stockage. Cette évaluation de l'acceptabilité 
d'un matériau alternatif pour un usage en technique routière nécessite une vraie connaissance sur la nature du déchet, le mode d'élaboration du matériau alternatif et du matériau routier comme le type d'usage routier envisagé et enfin l'évaluation environnementale des matériaux élaborés. L’objectif de la caractérisation environnementale est de montrer que les rejets éventuels des matériaux alternatifs et routiers respectent les objectifs de qualité des eaux. Cette caractérisation envisage trois niveaux d’investigations permettant de montrer le risque que présentent les matériaux alternatifs vis-à-vis de l'environnement :

- Le niveau 1 permet de fournir des informations sur la variabilité et justifier l'acceptabilité en technique routière des matériaux sur la base d'essais de lixiviation et d'analyse en contenu total.

- Le niveau 2 permet de justifier l'acceptabilité en technique routière sur la base d'essais de percolation si le niveau 1 n'est pas validé.

- Le niveau 3 se base sur une étude spécifique si les conditions1 et 2 n’ont pu être validées.

\section{Application à un sédiment du port de Dunkerque}

Une compagne de prélèvement en mars 2011 a été réalisée, les sédiments marins auxquels ils font objet de cette étude ont été prélevés à 0.5 mètre de profondeur par rapport à la surface dans un bassin à l'avant port Est DARSE 6 "Freycinet 12 et 13". Ces sédiments sont remplis et conservés dans 12 fûts en plastique hermétiques de 54 litres. Après la phase de prélèvement, une homogénéisation de tous les fûts a été réalisé dans un grand bac afin d'obtenir un matériau représentatif. Afin d'évaluer l'impact environnemental des sédiments marins pour une utilisation en technique routière, un test de lixiviation NF EN 12457-2 (AFNOR, 2002) a été réalisé selon le niveau 1 du guide. Le test consiste à évaluer la fraction mobilisable et la quantité de polluants relargués. Un mélange constitué de $1000 \mathrm{ml}$ d'eau déminéralisée et 100 g de sédiment marin sec ( $\mathrm{L} / \mathrm{S}=10)$ a subi une agitation de 60 cycles/ min pendant 24 heures. Après ce cycle nous avons séparé le lixiviat de la phase solide par centrifugation puis filtration sur un filtre de $0.45 \mu \mathrm{m}$. Pour évaluer la reproductibilité trois analyses ont été réalisées. Les résultats du test ainsi que les seuils définis par le guide méthodologique sont rassemblés dans le Tableau 1.

Vis-à-vis les valeurs limites associées à la caractérisation environnementale du niveau 1 fixé par le guide méthodologique, le relargage de tous les métaux mesurés dans notre étude est inférieur aux valeurs seuils. Par rapport aux limites à respecter pour 100\% des échantillons, l'écart est très important sauf pour le chlorure qui présente une valeur supérieure au seuil, d'autre part pour les limites à respecter pour une utilisation en technique routière les résultats sont inférieur aux seuils. Ce ci ne bloquerait pas le processus pour pouvoir valider la valorisation. 
La connaissance de la Mer :

un vecteur du développement durable en Méditerranée

\section{Conclusion}

L'évaluation des impacts environnementaux de sédiment marin de Dunkerque bassin Est DARSE 6 "FREYCINET 12 et 13" a été approchée au travers le test de lixiviation selon le guide méthodologique "acceptabilité de matériaux alternatifs en techniques routières, évaluation environnementale" du MEDDTL (SETRA, 2011). Au regard des résultats obtenus on estime que le sédiment marin se classe dans le niveau 1 du guide.

Tableau 1. Dosages des polluants sur les éluats.

\begin{tabular}{|c|c|c|c|c|}
\hline Éléments & $\begin{array}{l}\text { Valeur en } \\
\mathrm{mg} / \mathrm{kg} \text { de } \\
\text { matière sèche }\end{array}$ & $\begin{array}{l}\text { Valeur à respecter par } \\
\text { au moins } 100 \% \text { des } \\
\text { échantillons }(\mathrm{mg} / \mathrm{kg} \text { de } \\
\text { matière sèche) }{ }^{(* *)}\end{array}$ & $\begin{array}{l}\text { Valeurs limites de } \\
\text { lixiviation pour } \\
\text { une utilisation en } \\
\text { technique routière }\end{array}$ & Validation \\
\hline As & $<0,04$ & 1,5 & 2 & Oui \\
\hline$B a$ & 0,043 & 60 & 100 & Oui \\
\hline$C d$ & 0,004 & 0,12 & 1 & Oui \\
\hline $\mathrm{Cr}$ & 0,04 & 1,5 & 10 & Oui \\
\hline $\mathrm{Cu}$ & 0,05 & 6 & 50 & Oui \\
\hline $\mathrm{Hg}$ & $<0.05$ & 0,03 & 0.2 & Oui \\
\hline Mo & 0.092 & 1.5 & 10 & Oui \\
\hline $\mathrm{Ni}$ & 0.03 & 1.2 & 10 & Oui \\
\hline $\mathrm{Pb}$ & 0.04 & 1.5 & 10 & Oui \\
\hline$S b$ & 0.018 & 0.18 & 0.7 & Oui \\
\hline Se & 0.01 & 0.3 & 0.5 & Oui \\
\hline$Z n$ & 0.05 & 12 & 50 & Oui \\
\hline Fluorure & 0.61 & 30 & 150 & Oui \\
\hline Chlorure $^{(*)}$ & 5671 & 2400 & 15000 & Oui ${ }^{(*)}$ \\
\hline Sulfate ${ }^{(*)}$ & 871 & 3000 & 20000 & Oui \\
\hline Fraction soluble ${ }^{(*)}$ & 6440 & 12000 & 60000 & Oui \\
\hline$p H$ & 8.6 & Min 5.5/Max 13 & - & Oui \\
\hline COT & 23 & 60000 & - & Oui \\
\hline$P C B$ & $<0.01$ & 1 & - & Oui \\
\hline BTEX (Benzène toluène) & $<1$ & 6 & - & Oui \\
\hline \multicolumn{5}{|l|}{ HCT (hydrocarbure totaux, } \\
\hline C10 à C40) & $<200$ & 500 & - & Oui \\
\hline
\end{tabular}

Remarque:

La norme présente aussi d'autres valeurs seuils que celles reportées ici, que $80 \%$ ou $95 \%$ des échantillons doivent vérifier. Pour pouvoir les utiliser, il aurait fallu faire ces essais sur un nombre plus important d'échantillons. C'est pourquoi seules les valeurs seuils "Valeur à respecter par au moins 100\% des échantillons" et les "Valeurs limites de lixiviation pour une utilisation en technique routière" sont comparées avec nos résultats.

(*) Concernant les chlorures, les sulfates et la fraction soluble, il convient, pour être jugé conforme de respecter soit les valeurs associées aux chlorures et aux sulfates, soit de respecter les valeurs associées à la fraction soluble (SETRA, 2011).

$\left(^{* *}\right)$ Valeurs limites en lixiviation permettant de justifier toutes les utilisations visées par le guide méthodologique. 
Cette caractérisation environnementale permet au final de graduer l'effort de démonstration en fonction du risque que représente le sédiment vis-à-vis de l'environnement. L'analyse environnementale a été menée dans le cadre d'une valorisation routière envisagée. Cependant le sédiment du port de Dunkerque présente une teneur en eau initiale élevée et une plasticité importante dû à la présence de la fraction argileuse et de la matière organique. Il sera donc nécessaire de faire subir un prétraitement à fin de réduire la teneur en eau et améliorés leurs caractéristiques mécaniques.

\section{Références}

AFNOR -Association Française de Normalisation- (2002). NF EN 12457-2 : Caractérisation des déchets : lixiviation - Essai de conformité pour lixiviation des déchets fragmentés et des boues. Partie 2 : essai en bâchée unique $(L / S=10)$.

BOUTIN R. (2000). Dragage et rejets en mer: les produits de type vase. Presses de l'école nationale des ponts et chaussées, 307 p.

CETMEF (2010). Enquête dragage 2008 : analyse des données. juillet 2010

DUBOIS V., ABRIAK N.E., ZENTAR R., BALLIVY G. (2009). The use of marine sediments as a pavement base material. Waste Management 29(2), pp 774-82. doi:10.1016/j.wasman.2008.05.004

UNPG -Union nationale des producteurs de granulats- (site web). Portrait économique. http://www.unpg.fr/dossiers/economie/portrait_economique

SETRA -Service technique du Ministère de l'Ecologie, du Développement durable, des Transports et du Logement- (2011). Guide méthodologique acceptabilité de matériaux alternatifs en technique routière. $32 \mathrm{p}$. 
La connaissance de la Mer :

un vecteur du développement durable en Méditerranée 